\title{
541.
}

\section{ON THE RECIPROCAL OF A CERTAIN EQUATION OF A CONIC.}

[From the Messenger of Mathematics, vol. I. (1872), pp. 120, 121.]

THE following formula is useful in various problems relating to conics: the reciprocal equation of the conic

$$
\lambda(a x+b y+c z)\left(a^{\prime} x+b^{\prime} y+c^{\prime} z\right)-\mu\left(a^{\prime \prime} x+b^{\prime \prime} y+c^{\prime \prime} z\right)\left(a^{\prime \prime \prime} x+b^{\prime \prime \prime} y+c^{\prime \prime \prime} z\right)=0
$$

may be written indifferent in either of the forms

$$
\left\{\lambda\left|\begin{array}{ccc}
\xi, & \eta, & \zeta \\
a^{\prime}, & b^{\prime}, & c^{\prime} \\
a, & b, & c
\end{array}\right|+\mu\left|\begin{array}{ccc}
\xi, & \eta, & \zeta \\
a^{\prime \prime}, & b^{\prime \prime}, & c^{\prime \prime} \\
a^{\prime \prime \prime}, & b^{\prime \prime \prime}, & c^{\prime \prime \prime}
\end{array}\right|\right\}^{2}+4 \lambda \mu\left|\begin{array}{lll}
\xi, & \eta, & \zeta \\
a, & b, & c \\
a^{\prime \prime \prime}, & b^{\prime \prime \prime}, & c^{\prime \prime \prime}
\end{array}\right|\left|\begin{array}{ccc}
\xi, & \eta, & \zeta \\
a^{\prime}, & b^{\prime}, & c^{\prime} \\
a^{\prime \prime}, & b^{\prime \prime}, & c^{\prime \prime}
\end{array}\right|=0,
$$

and

$$
\left\{\lambda\left|\begin{array}{ccc}
\xi, & \eta, & \zeta \\
a^{\prime}, & b^{\prime}, & c^{\prime} \\
a, & b, & c
\end{array}\right|-\mid \begin{array}{ccc}
\xi, & \eta, & \zeta \\
a^{\prime \prime}, & b^{\prime \prime}, & c^{\prime \prime} \\
a^{\prime \prime \prime}, & b^{\prime \prime \prime}, & c^{\prime \prime \prime}
\end{array}\right\}^{2}+4 \lambda \mu\left|\begin{array}{lll}
\xi, & \eta, & \zeta \\
a, & b, & c \\
a^{\prime \prime}, & b^{\prime \prime}, & c^{\prime \prime}
\end{array}\right|\left|\begin{array}{ccc}
\xi, & \eta, & \zeta \\
a^{\prime}, & b^{\prime}, & c^{\prime} \\
a^{\prime \prime \prime}, & b^{\prime \prime \prime}, & c^{\prime \prime \prime}
\end{array}\right|=0 .
$$

In fact, in the reciprocal equation, seeking for the coefficient of $\xi^{2}$, it is

$$
\left\{\lambda\left(b c^{\prime}+b^{\prime} c\right)-\mu\left(b^{\prime \prime} c^{\prime \prime \prime}+b^{\prime \prime \prime} c^{\prime \prime}\right)\right\}^{2}-\left(2 \lambda b b^{\prime}-2 \mu b^{\prime \prime} b^{\prime \prime \prime}\right)\left(2 \lambda c c^{\prime}-2 \mu c^{\prime \prime} c^{\prime \prime \prime}\right),
$$

viz. this is

$$
\lambda^{2}\left(b c^{\prime}-b^{\prime} c\right)^{2}+\mu^{2}\left(b^{\prime \prime} c^{\prime \prime \prime}-b^{\prime \prime \prime} c^{\prime \prime}\right)^{2}+2 \lambda \mu\left\{\begin{array}{c}
2 b b^{\prime} c^{\prime \prime} c^{\prime \prime \prime}+2 b^{\prime \prime} b^{\prime \prime \prime} c c^{\prime} \\
-\left(b c^{\prime}+b^{\prime} c\right)\left(b^{\prime \prime} c^{\prime \prime \prime}+b^{\prime \prime \prime} c^{\prime \prime}\right)
\end{array}\right\}
$$


or, as it may be written,

$$
\left\{\lambda\left(b c^{\prime}-b^{\prime} c\right) \pm \mu\left(b^{\prime \prime} c^{\prime \prime \prime}-b^{\prime \prime \prime} c^{\prime \prime}\right)\right\}^{2}+2 \lambda \mu\left\{\begin{array}{c}
2 b b^{\prime} c^{\prime \prime} c^{\prime \prime \prime}+2 b^{\prime \prime} b^{\prime \prime \prime} c c^{\prime} \\
-\left(b c^{\prime}+b^{\prime} c\right)\left(b^{\prime \prime} c^{\prime \prime \prime}+b^{\prime \prime \prime} c^{\prime \prime}\right) \\
\mp\left(b c^{\prime}-b^{\prime} c\right)\left(b^{\prime \prime} c^{\prime \prime \prime}-b^{\prime \prime \prime} c^{\prime \prime}\right)
\end{array}\right\} .
$$

Taking the upper signs, this is

viz. the term in $\lambda \mu$ is

$$
\left\{\lambda\left(b c^{\prime}-b^{\prime} c\right)+\mu\left(b^{\prime \prime} c^{\prime \prime \prime}-b^{\prime \prime \prime} c^{\prime \prime}\right)\right\}^{2}+4 \lambda \mu\left(\begin{array}{r}
b b^{\prime} c^{\prime \prime} c^{\prime \prime \prime}+b^{\prime \prime} b^{\prime \prime \prime} c c^{\prime} \\
-b c^{\prime} b^{\prime \prime} c^{\prime \prime \prime}-b^{\prime} c b^{\prime \prime \prime} c^{\prime \prime}
\end{array}\right),
$$

$$
+4 \lambda \mu\left(b c^{\prime \prime \prime}-b^{\prime \prime \prime} c\right)\left(b^{\prime} c^{\prime \prime}-b^{\prime \prime} c^{\prime}\right)
$$

Taking the lower signs, it is

$$
\left\{\lambda\left(b c^{\prime}-b^{\prime} c\right)-\mu\left(b^{\prime \prime} c^{\prime \prime \prime}-b^{\prime \prime \prime} c^{\prime \prime}\right)\right\}^{2}+4 \lambda \mu\left(\begin{array}{r}
b b^{\prime} c^{\prime \prime} c^{\prime \prime \prime}+b^{\prime \prime} b^{\prime \prime \prime} c c^{\prime} \\
-b c^{\prime} b^{\prime \prime \prime} c^{\prime \prime}-b^{\prime} c b^{\prime \prime} c^{\prime \prime \prime}
\end{array}\right),
$$

viz. the term in $\lambda \mu$ is

$$
+4 \lambda \mu\left(b c^{\prime \prime}-b^{\prime \prime} c\right)\left(b^{\prime} c^{\prime \prime \prime}-b^{\prime \prime \prime} c^{\prime}\right)
$$

And it is thence easy to infer the forms of the other coefficients, and to arrive at the foregoing result. 